\title{
Fault Tolerant Control for Over-Actuated Systems: An Adaptive Correction Approach
}

\author{
Seyed Shahabaldin Tohidi ${ }^{1}$, Yildiray Yildiz ${ }^{1}$ and Ilya Kolmanovsky ${ }^{2}$
}

\begin{abstract}
This paper proposes an adaptive fault tolerant control allocation approach for over-actuated systems. The methodology does not utilize the control input matrix estimation to tolerate actuator faults and, therefore, the proposed control allocation method does not require persistence of excitation. Adaptive control approach with a closed loop reference model is used for identifying control allocation parameters, which provides improved performance without introducing undesired oscillations. Furthermore, a sliding mode controller is used to guarantee the outer loop asymptotic stability. Simulation results are provided, where the ADMIRE model is used as an over-actuated system, to demonstrate the effectiveness of the proposed method.
\end{abstract}

\section{INTRODUCTION}

Actuator faults reduce the performance of the system and may cause catastrophic accidents. A common approach to address actuator faults is to introduce actuator redundancy and to manage control signals among redundant actuators by utilizing control allocation methodologies.

Surveys on control allocation methodologies and various methods of reconfigurable fault tolerant control can be found in [1] and [2], respectively. Two main control allocation methodologies that are used for fault tolerance applications are optimization based control allocation and adaptive control allocation.

One example of optimization based control allocation methods is given in [3], where error minimization is used to improve the performance of steering in automotive vehicles considering faults as asphalt conditions that should be estimated. In another study, thruster force in a faulty underwater vehicle is allocated among redundant thrusters using control minimization [4]. This method is also implemented on a modified quad-rotor helicopter in [5] where the experimental results under different propeller faults are presented. Optimization based control allocation for fault tolerance applications is implemented in various other overactuated systems, where in the majority of the cases, the control input matrix is either estimated or assumed to be estimated [6]-[11].

Adaptive control allocation methods have low computational complexity in comparison with optimization based control allocation methods. However, these methods require persistence of excitation and may exhibit oscillatory behavior

\footnotetext{
${ }^{1}$ Seyed Shahabaldin Tohidi and Yildiray Yildiz are with Faculty of Mechanical Engineering, Bilkent University, Cankaya, Ankara 06800, Turkey shahabaldin@bilkent.edu.tr, yyildiz@bilkent.edu.tr

${ }^{2}$ Ilya Kolmanovsky is with the Department of Aerospace Engineering, University of Michigan, Ann Arbor, MI 48109, USA i lya@umi ch . edu
}

during parameter estimation. In [6], faults are estimated adaptively and a methodology is proposed in order to obtain persistence of excitation. The control allocation problem is considered as a gain scheduling problem in [12] and the gains are estimated adaptively, where the allocation problem is coupled with the model reference adaptive controller design. A general adaptive fault tolerant controller is proposed in [13] where, the actuator lock-in-place failures are tolerated using adaptive state feedback. Fault detection and isolation methodologies are other useful methods that can provide estimated fault information for control allocation (see [14]). In [15], an unknown input observer is applied to identify actuator and effector faults. Sliding mode controller is coupled with control allocation to design a fault tolerant controller in [16], where the faults are assumed to be estimated.

This paper proposes a new control allocation method that can adaptively tolerate faults in systems with actuator redundancies. The method does not need fault estimation, so it does not require persistence of excitation or additional sensors to determine actuator effectiveness. Control allocation parameters are estimated rapidly without causing excessive oscillations with the help of the adaptive method that utilizes closed loop reference models [17]. In addition, a sliding mode control is designed to control the outer loop and guarantee the stability and reference tracking.

This paper is organized as follow. Section II presents the faulty over-actuated system where actuator faults are modeled as loss of effectiveness. The adaptive control allocation is presented in Section III. Section IV presents the sliding mode controller design. The ADMIRE model is used in Section V to illustrate the effectiveness of the proposed methodology in the simulation environment. Finally, Section VI concludes the paper.

It is noted that throughout the paper, Frobenius norm is used.

\section{PROBLEM STATEMENT}

Consider the following plant dynamics

$$
\begin{aligned}
\dot{x} & =A x+B_{u} u \\
& =A x+B_{v} B u .
\end{aligned}
$$

where $x \in R^{n}$ is the system states vector, $u \in R^{m}$ is the control input vector, $A \in R^{n \times n}$ is the known state matrix and $B_{u} \in R^{n \times m}$ is the known control input matrix which is decomposed into the known matrices $B_{v} \in R^{n \times r}$ and $B \in R^{r \times m}$. To model the actuator degradation, a diagonal 
matrix $\Lambda \in R^{m \times m}$ with uncertain positive elements is added to the system dynamics as follows

$$
\begin{aligned}
\dot{x} & =A x+B_{v} B \Lambda u \\
& =A x+B_{v} v
\end{aligned}
$$

where $v \in R^{r}$ denotes the virtual control input produced by the outer loop controller.

The control allocation problem is to achieve

$$
B \Lambda u=v
$$

Since $\Lambda$ is unknown, conventional control allocation methods do not apply here. In addition, it is required that matrix identification methods are not used since they require persistence of excitation which is hard to realize in real applications.

\section{ADAPTIVE CONTROL ALLOCATION}

In this section, we develop the proposed adaptive control allocation method. Towards this end, we first transform the control allocation problem into a conventional model reference adaptive control problem and then develop the corresponding adaptive laws.

Consider the following dynamics

$$
\dot{y}=A_{m} y+B \Lambda u-v
$$

where $A_{m} \in R^{r \times r}$ is stable matrix and a reference model given by

$$
\dot{y}_{m}=A_{m} y_{m} .
$$

Defining the control input as a mapping from $v$ to $u$,

$$
u=\theta_{v}^{T} v
$$

where $\theta_{v} \in R^{r \times m}$ represents the adaptive parameter matrix to be determined, and substituting (6) into (4), we obtain that

$$
\dot{y}=A_{m} y+\left(B \Lambda \theta_{v}^{T}-I\right) v .
$$

It is assumed that there exists a $\theta_{v}^{*}$ such that

$$
B \Lambda \theta_{v}^{* T}=I .
$$

Defining $\theta_{v}^{T}=\theta_{v}^{* T}+\tilde{\theta}_{v}^{T}$, where $\tilde{\theta}_{v}^{T}$ is the deviation of $\theta_{v}^{T}$ from its ideal value, equation (7) can be rewritten as

$$
\dot{y}=A_{m} y+B \Lambda \tilde{\theta}_{v}^{T} v .
$$

Defining an error $e=y-y_{m}$, and taking its derivative using (5) and (9), it follows that

$$
\dot{e}=A_{m} e+B \Lambda \tilde{\theta}_{v}^{T} v .
$$

Let $\Gamma=\Gamma^{T}=\gamma I_{r} \in R^{r \times r}>0$, where $\gamma$ is a positive scalar, and consider a Lyapunov function candidate

$$
V=e^{T} P e+\operatorname{tr}\left(\tilde{\theta}_{v}^{T} \Gamma^{-1} \tilde{\theta}_{v} \Lambda\right),
$$

where $t r$ refers to the trace operation and $P$ is the positive definite symmetric matrix solution of the Lyapunov equation

$$
A_{m}^{T} P+P A_{m}=-Q
$$

where $Q$ is a symmetric positive definite matrix. The derivative of the Lyapunov function candidate can be calculated as

$$
\begin{aligned}
\dot{V}= & e^{T}\left(A_{m}^{T} P+P A_{m}\right) e+2 e^{T} P B \Lambda \tilde{\theta}_{v}^{T} v \\
& +2 \operatorname{tr}\left(\tilde{\theta}_{v}^{T} \Gamma^{-1} \dot{\tilde{\theta}}_{v} \Lambda\right) \\
= & -e^{T} Q e+2 e^{T} P B \Lambda \tilde{\theta}_{v}^{T} v+2 \operatorname{tr}\left(\tilde{\theta}_{v}^{T} \Gamma^{-1} \dot{\tilde{\theta}}_{v} \Lambda\right) .
\end{aligned}
$$

Using the property of the trace operation $a^{T} b=\operatorname{tr}\left(b a^{T}\right)$ where $a$ and $b$ are vectors, (13) can be rewritten as

$$
\dot{V}=-e^{T} Q e+2 \operatorname{tr}\left(\tilde{\theta}_{v}^{T}\left(v e^{T} P B+\Gamma^{-1} \dot{\tilde{\theta}}_{v}\right) \Lambda\right) .
$$

It is shown below that the following adaptive law can be used to obtain $\dot{V} \leq 0$.

$$
\dot{\theta}_{v}=\Gamma \operatorname{Proj}\left(\theta_{v},-v e^{T} P B\right)
$$

where "Proj" refers to the following projection operator: Consider $Y=-v e^{T} P B \in R^{r \times m}$ and $\theta_{v} \in R^{r \times m}$,

$$
\operatorname{Proj}\left(\theta_{v}, Y\right)=\left(\operatorname{Proj}\left(\theta_{v, 1}, Y_{1}\right), \ldots, \operatorname{Proj}\left(\theta_{v, m}, Y_{m}\right)\right)
$$

where $\theta_{v, i}$ and $Y_{i}$ refer to the $i$ th column of $\theta_{v}$ and $Y$ respectively, and

$$
\begin{aligned}
& \operatorname{Proj}\left(\theta_{v, i}, Y_{i}\right) \equiv \\
& \left\{\begin{array}{c}
Y_{i}-\frac{\nabla f_{i}\left(\theta_{v, i}\right)\left(\nabla f_{i}\left(\theta_{v, i}\right)\right)^{T}}{\left\|\nabla f_{i}\left(\theta_{v, i}\right)\right\|^{2}} Y_{i} f_{i}\left(\theta_{v, i}\right) \quad \text { if } f_{i}\left(\theta_{v, i}\right)>0 \\
Y_{i} \quad \& Y_{i}^{T} \nabla f_{i}\left(\theta_{v, i}\right)>0
\end{array}\right.
\end{aligned}
$$

where $\nabla$ is the gradient operator and $f_{i}$ is a convex vector function defined as

$$
f_{i}=f\left(\theta_{v, i}\right)=\frac{\left\|\theta_{v, i}\right\|^{2}-\theta_{\max , i}^{* 2}}{\varepsilon_{i} \theta_{\max , i}^{* 2}}
$$

where $\varepsilon_{i}>0$ is the projection tolerance of the $i$ th column of $\theta_{v}$ and $\theta_{\max , i}^{*}$ is a known upper bound such that $\left\|\theta_{v, i}^{*}\right\| \leq \theta_{\max , i}^{*}$. In the convex function (18), $f\left(\theta_{v, i}\right)=0$ when $\left\|\theta_{v, i}\right\|=\theta_{\max , i}^{*}$ and $f\left(\theta_{v, i}\right)=1$ when $\left\|\theta_{v, i}\right\|=$ $\theta_{\max , i}^{*} \sqrt{1+\varepsilon_{i}}$.

Lemma 1 [22]: If an adaptive algorithm with adaptive law $\dot{\theta}_{v, i}=\operatorname{Proj}\left(\theta_{v, i}, Y_{i}\right)$ and initial conditions $\theta_{v, i}(0) \in \Omega_{i}=$ $\left\{\theta_{v, i} \in R^{r} \mid f\left(\theta_{v, i}\right) \leq 1\right\}$ and a convex function $f\left(\theta_{v, i}\right)$ : $R^{r} \rightarrow R$ is defined, then $\theta_{v, i} \in \Omega_{i}$ for $\forall t \geq 0$.

Using the inequality $\left\|\tilde{\theta}_{v}\right\| \leq\left\|\theta_{v}\right\|+\left\|\theta_{v}^{*}\right\|$, it is obtained that $\left\|\tilde{\theta}_{v, i}\right\| \leq \tilde{\theta}_{\max , i}=\theta_{\max , i}^{*}\left(1+\sqrt{1+\varepsilon_{i}}\right)$.

Substituting (15) into (14), the derivative of the Lyapunov function candidate is obtained as

$$
\begin{aligned}
& \dot{V}=-e^{T} Q e \\
& +2 \operatorname{tr}\left(\tilde{\theta}_{v}^{T}\left(v e^{T} P B+\operatorname{Proj}\left(\theta_{v},-v e^{T} P B\right)\right) \Lambda\right)
\end{aligned}
$$

By using the property of the projection operator given in [21]:

$$
\operatorname{tr}\left(\tilde{\theta}_{v}^{T}\left(-Y+\operatorname{Proj}\left(\theta_{v}, Y\right)\right) \Lambda\right) \leq 0
$$

we obtain that $\dot{V} \leq 0$. 
A negative semi-definite Lyapunov function derivative ensures that the error signal $e$ and the adaptive parameter $\theta_{v}$ are bounded. For the initial conditions $e(0)$ and $\left\|\theta_{v, i}(0)\right\| \leq$ $\theta_{\text {max }, i}^{*} \sqrt{1+\varepsilon_{i}}, e$ and $\theta_{v}$ are uniformly bounded for $\forall t \geq 0$ and system trajectories converge to the following set [22]

$$
E=\left\{\left(e, \tilde{\theta}_{v}\right):\|e\|^{2} \leq \frac{2 s \tilde{\theta}_{\max }^{2}}{\gamma},\left\|\tilde{\theta}_{v, i}\right\| \leq \tilde{\theta}_{\max , i}\right\}
$$

where

$$
\begin{array}{r}
s \equiv-\min _{i}\left(\lambda_{i}\left(A_{m}+A_{m}^{T}\right) / 2\right) \\
\Gamma^{-1} \equiv \frac{1}{\gamma} I_{r}
\end{array}
$$

It is already shown that $\tilde{\theta}_{v, i} \leq \theta_{\text {max }, i}^{*}\left(1+\sqrt{1+\varepsilon_{i}}\right)$, therefore

$$
\begin{aligned}
\left\|\tilde{\theta}_{v}\right\| & =\sqrt{\sum_{i=1}^{m} \sum_{j=1}^{r}\left|\tilde{\theta}_{v_{i, j}}\right|^{2}}=\sqrt{\sum_{i=1}^{m} \|\left.\tilde{\theta}_{v_{i}}\right|^{2}} \\
& \leq \sqrt{\sum_{i=1}^{m} \theta_{\max , i}^{* 2}\left(1+\sqrt{1+\varepsilon_{i}}\right)^{2}} \equiv \tilde{\theta}_{\max }
\end{aligned}
$$

To obtain fast convergence without introducing excessive oscillations, the open loop reference model (5) is modified as a closed loop reference model as follows

$$
\dot{y}_{m}=A_{m} y_{m}-L\left(y-y_{m}\right)
$$

where $L=-l I_{r} \in R^{r \times r}$ and $l$ is a positive scalar design parameter. It is noted that the above stability analysis can be modified using this reference model. For details, see [17]. For the initial conditions $e(0)$ and $\left\|\theta_{v, i}(0)\right\| \leq \theta_{\max , i}^{*} \sqrt{1+\varepsilon_{i}}$, $e$ and $\theta_{v}$ are uniformly bounded for $\forall t \geq 0$ and system trajectories converge to the following set [22]

$$
E=\left\{\left(e, \tilde{\theta}_{v}\right):\|e\|^{2} \leq \frac{2(s+l) \tilde{\theta}_{\max }^{2}}{\gamma},\left\|\tilde{\theta}_{v, i}\right\| \leq \tilde{\theta}_{\max , i}\right\}
$$

\section{OUTER LOOP CONTROLLER DESIGN IN THE PRESENCE OF CONTROL ALLOCATION ERROR}

Since the control allocation subsystem has limited bandwidth, the virtual control signal $v$, produced by the outer loop controller, and the achieved moments $B \Lambda u$ will not be the same instantaneously.

To reflect this fact, substituting (6) into (2), we rewrite the plant dynamics as

$$
\begin{aligned}
\dot{x} & =A x+B_{v} B \Lambda u \\
& =A x+B_{v} B \Lambda \theta_{v}^{T} v \\
& =A x+B_{v}\left(B \Lambda \theta_{v}^{* T}+B \Lambda \tilde{\theta}_{v}^{T}\right) v .
\end{aligned}
$$

Substituting the ideal value of $\theta_{v}^{*}$ in (17), such that $B \Lambda \theta_{v}^{* T}=$ $I$, we obtain that

$$
\dot{x}=A x+B_{v}\left(I+B \Lambda \tilde{\theta}_{v}^{T}\right) v
$$

Since the projection algorithm is used in the adaptive laws for the control allocation, we know that $\tilde{\theta}_{v}$ is bounded, regardless of any stability condition. Defining $F(t) \equiv B \Lambda \tilde{\theta}_{v}^{T}$, (26) can be rewritten as

$$
\dot{x}=A x+B_{v}(I+F(t)) v
$$

where $F(t) \in R^{r \times r}$ is a bounded function.

Lemma 2: There exists $\bar{F}$ such that $\|F(t)\| \leq \bar{F}, \forall t$, where $\bar{F}$ is a known constant.

Proof of lemma 2: Using $F(t)=B \Lambda \tilde{\theta}_{v}^{T}$, it is obtained that

$$
\|F(t)\| \leq\|B\| \sqrt{m} \tilde{\theta}_{\max }
$$

It is noted that the definition of $\tilde{\theta}_{\max }$ is given in (22).

Assume that (27) can be decomposed into two subsystems given as

$$
\begin{aligned}
& \dot{x}_{1}=A_{1} x_{1}+A_{2} x_{2} \\
& \dot{x}_{2}=A_{3} x_{1}+A_{4} x_{2}+B_{v}^{\prime}(I+F(t)) v
\end{aligned}
$$

where $x_{1} \in R^{n-r}, x_{2} \in R^{r}$. Furthermore, assume that $A_{1}$ is stable and $B_{v}^{\prime} \in R^{r \times r}$ is an invertible matrix. These conditions make (29) a square system which is suitable for the application of sliding mode control [18]. In addition, since $A_{1}$ is stable, showing that the states $x_{2}$ are bounded will be enough for the boundedness of $x_{1}$.

Each individual scalar equation in (29) can be written as

$$
\dot{x}_{2 i}=h_{i}(x)+\sum_{j=1}^{r} b_{i j}(x) v_{j} \quad i=1, \ldots, r, \quad j=1, \ldots, r
$$

Defining

$$
s_{i}=x_{2 i}-x_{2 d_{i}}
$$

where $x_{2 d_{i}}$ is the desired trajectory for $x_{2 i}$, it can be shown that the following control input satisfies the sliding conditions

$$
v=B_{v}^{\prime-1}\left(x_{2 d}-h(x)-k \operatorname{sgn}(s)\right),
$$

where $x_{2 d} \in R^{r}, h(x) \in R^{r}$ and $k \operatorname{sgn}(s) \in R^{r}$ is a vector consisting of components $k_{i} \operatorname{sgn}\left(s_{i}\right)$. It is noted that the elements of vector $k$ must be chosen such that

$$
(1-\bar{F}) k_{i}+\sum_{j \neq i} \bar{F} k_{j}=\sum_{j=1}^{r} \bar{F}\left|x_{2 d_{i}}-h_{j}\right|+\eta_{i}, \quad i=1, \ldots, r
$$

where $\eta_{i} \in R$ is the positive scalar used in the sliding condition given as

$$
\frac{1}{2} \frac{d}{d t} s_{i}^{2} \leq-\eta_{i}\left|s_{i}\right| .
$$

Chattering is an undesirable result of the controller (32). Using the boundary layer approach, one can smooth out the control discontinuity $(\operatorname{sgn}(s))$ near the switching surface. Instead of the term $\operatorname{sgn}(s)$, we use $\operatorname{sat}(s / \Phi)$ in order to avoid discontinuity in the controller design:

$$
v=B_{v}^{\prime-1}\left(x_{2 d}-h(x)+k \operatorname{sat}(s / \Phi)\right)
$$


where $\Phi$ is the boundary layer thickness and sat is the saturation function.. The sliding mode controller (35) guarantees that the boundary layer is attractive and invariant.

The state vector $x_{2}$ is bounded and using (28), it is concluded that $x_{1}$ is also bounded. Using (28) and (29), it is seen that $\dot{x}_{1}$ and $\dot{x}_{2}$ are bounded. Therefore, $v$ and $\dot{v}$ are bounded. This shows that the requirements for the stability analysis of the control allocation system are satisfied.

\section{APPLICATION EXAMPLE}

\section{A. ADMIRE MODEL}

The simplified ADMIRE model provided in [19], [20] is used in this section to demonstrate the effectiveness of the proposed method in the presence of actuator faults. The states, outputs and control deflections of the system are given as below

$$
\begin{aligned}
x & =\left[\begin{array}{llll}
\alpha & \beta & p & q \\
y
\end{array}\right]^{T} \\
y & =\left[\begin{array}{lll}
p & q & r
\end{array}\right]^{T} \\
u & =\left[\begin{array}{llll}
u_{c} & u_{r e} & u_{l e} & u_{r}
\end{array}\right]^{T}
\end{aligned}
$$

where $\alpha, \beta, p, q$ and $r$ are the angle of attack, sideslip angle, roll rate, pitch rate and yaw rate, respectively. Control surfaces are canard wings, right and left elevons and the rudder. The following approximate model provided in [19] represents the linearized aircraft dynamics at Mach 0.22 and altitude $3000 \mathrm{~m}$,

$$
\begin{gathered}
\dot{x}=A x+B_{u} u=A x+B_{v} v \\
v=B u, \quad B_{u}=B_{v} B, \quad B_{v}=\left[\begin{array}{c}
0_{2 \times 3} \\
I_{3 \times 3}
\end{array}\right]
\end{gathered}
$$

where state and control matrices are as below

$$
\begin{gathered}
{\left[\begin{array}{ccccc}
-0.5432 & 0.0137 & 0 & 0.9778 & 0 \\
0 & -0.1179 & 0.2215 & 0 & -0.9661 \\
0 & -10.5123 & -0.9967 & 0 & 0.6176 \\
2.6221 & -0.0030 & 0 & -0.5057 & 0 \\
0 & 0.7075 & -0.0939 & 0 & -0.2127
\end{array}\right]} \\
B=\left[\begin{array}{cccc}
0 & -4.2423 & 4.2423 & 1.4871 \\
1.6532 & -1.2735 & -1.2735 & 0.0024 \\
0 & -0.2805 & 0.2805 & -0.8823
\end{array}\right]
\end{gathered}
$$

In order to provide actuator redundancy in a form that can be exploited using control allocation, the control surfaces are viewed as pure moment generators and their influence on derivatives of the first two states i.e. $\dot{\alpha}$ and $\dot{\beta}$ is neglected [20]. In addition, to represent actuator faults, a diagonal matrix $\Lambda$ is introduced. As discussed in the previous section, this system can be written as two subsystems:

$$
\begin{aligned}
& {\left[\begin{array}{c}
\dot{\alpha} \\
\dot{\beta}
\end{array}\right]=\left[\begin{array}{cc}
-0.5432 & 0.0137 \\
0 & -0.1179
\end{array}\right]\left[\begin{array}{l}
\alpha \\
\beta
\end{array}\right]} \\
& +\left[\begin{array}{ccc}
0 & 0.9778 & 0 \\
0.2215 & 0 & -0.9661
\end{array}\right]\left[\begin{array}{l}
p \\
q \\
r
\end{array}\right]
\end{aligned}
$$

$$
\begin{aligned}
& {\left[\begin{array}{c}
\dot{p} \\
\dot{q} \\
\dot{r}
\end{array}\right]=\left[\begin{array}{cc}
0 & -10.5123 \\
2.6221 & -0.0030 \\
0 & 0.7075
\end{array}\right]\left[\begin{array}{l}
\alpha \\
\beta
\end{array}\right] } \\
+ & {\left[\begin{array}{ccc}
-0.9967 & 0 & 0.6176 \\
0 & -0.5057 & 0 \\
-0.0939 & 0 & -0.2127
\end{array}\right]\left[\begin{array}{l}
p \\
q \\
r
\end{array}\right] } \\
+ & B_{v}^{\prime}(I+F(t)) v
\end{aligned}
$$

where $B_{v}^{\prime}$ is the identity matrix and $(I+F(t)) v=B \Lambda u$. Since the ADMIRE model is written in the form of (28) and (29), the proposed sliding mode controller can be applied to this system.

The closed loop reference model provided in (23) is used with $l=4$ and $A_{m}$ selected as

$$
A_{m}=-\left[\begin{array}{ccc}
0.2 & 0 & 0 \\
0 & 0.1 & 0 \\
0 & 0 & 0.1
\end{array}\right]
$$

For simulations, we have assumed that all actuators are healthy in the first 10 seconds of the simulation and their effectiveness are reduced afterwards. The uncertainty matrix used in the simulations is given as

$$
\Lambda(t)= \begin{cases}\operatorname{diag}(1,1,1,1) & \text { for } t<10(\mathrm{sec}) \\ \operatorname{diag}(0.8,0.8,0.8,0.8) & \text { for } t \geq 10(\mathrm{sec})\end{cases}
$$

The elements of the vector $k$ are calculated using (33).

\section{B. Simulation Results}

Figure 1 shows the adaptation parameters. It is seen that all of the parameters are bounded. Figure 2 shows that the states $\alpha$ and $\beta$ remain bounded while $p, q$ and $r$ follow their desired references. $p, q$ and $r$ continue to track their desired values after the fault occurs at $t=10 \mathrm{sec}$. Figure 3 and Figure 4 show the components of the virtual control input and the control surface deflections, respectively. It is seen from the figures that control allocation successfully follows the virtual control inputs and corresponding control surface deflections remain bounded with smooth variations.
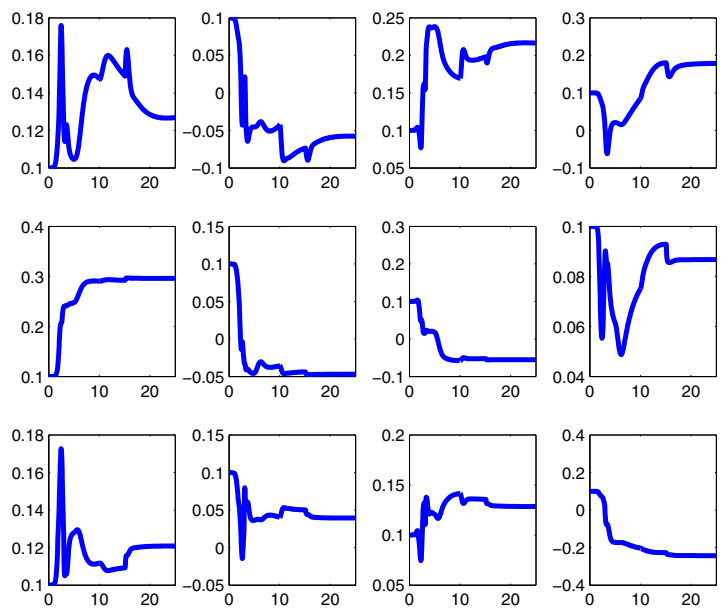

Fig. 1: Adaptation parameters (elements of $\theta_{v} \in R^{3 \times 4}$ ). 

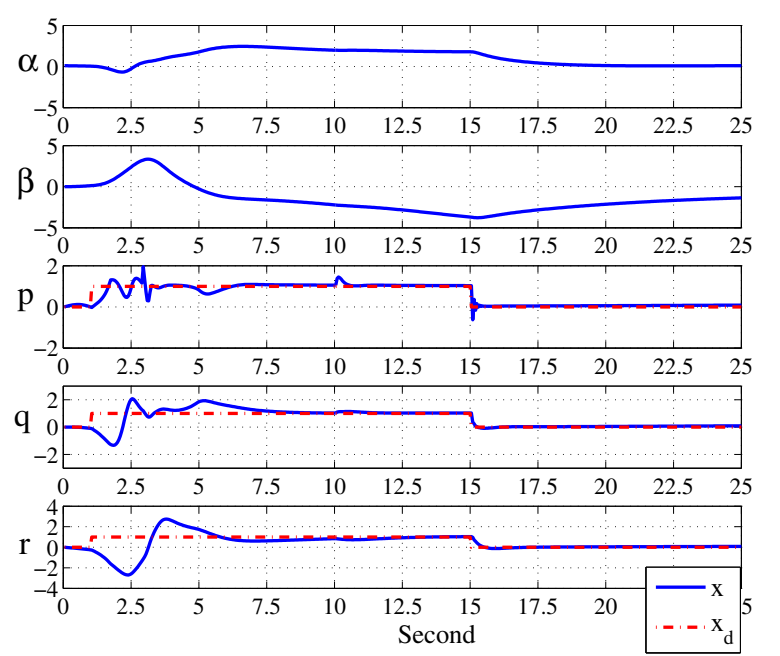

Fig. 2: System states.
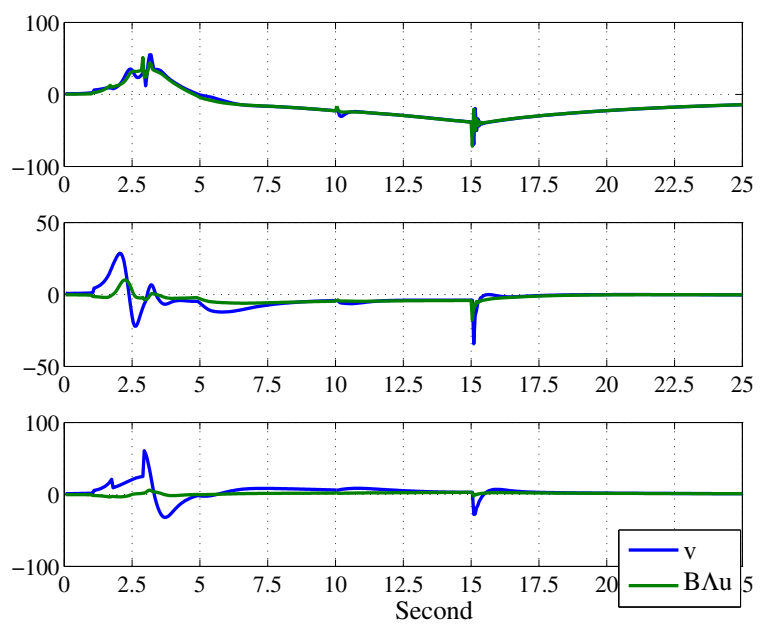

Fig. 3: Virtual control signal $v$ tracking.

\section{CONCLUSIONS}

An adaptive fault tolerant controller using control allocation is proposed in this paper. This method does not need fault identification and does not require persistence excitation conditions to achieve convergence. Using closed loop reference models improves the performance of the adaptive control allocation without causing excessive oscillations. The proposed adaptive control allocation method has modular design, allowing the flexibility to develop the outer loop controller and the control allocation strategy separately. A sliding mode controller is utilized as the outer loop controller to compensate the transient tracking error of the control allocator. The simulation results of the ADMIRE model show that the proposed adaptive control allocator achieves the tracking of virtual control inputs. In addition, the overall structure, together with the outer loop sliding mode controller, is stable and the plant outputs follow their references in the presence of actuator faults.
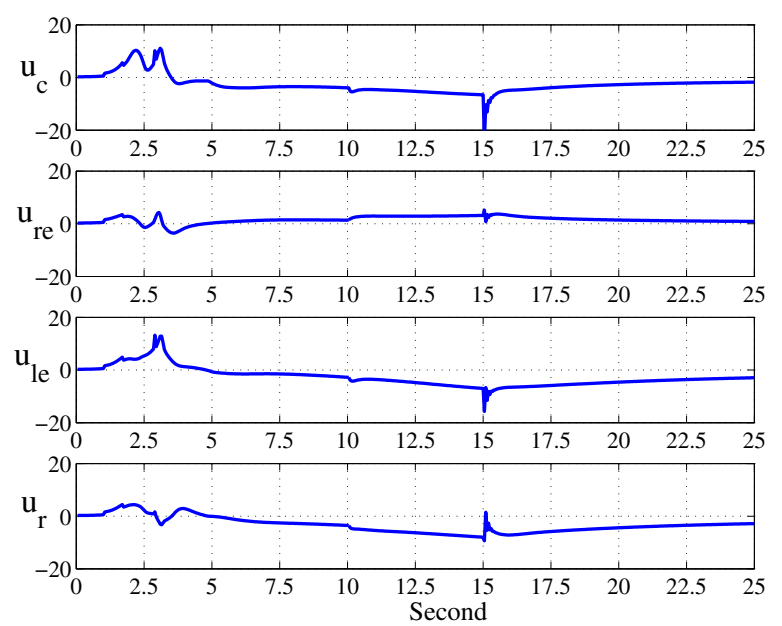

Fig. 4: Control surface deflection $u$ elements.

\section{ACKNOWLEDGEMENTS}

Author Yildiray Yildiz would like to thank the Scientific and Technological Research Council of Turkey (TUBITAK) for its financial support through the 2232 Reintegration Scholarship Program.

\section{REFERENCES}

[1] T. A. Johansen, and T. I. Fossen, "Control allocation-a survey," Automatica, vol. 49, pp. 1087-1103, May 2013.

[2] Y. Zhang, "Bibliographical review on reconfigurable fault-tolerant control systems," Annual reviews in control, vol. 32, pp. 229-252, Dec. 2008.

[3] J. Tjonnas, and T. A. Johansen, "Stabilization of automotive vehicles using active steering and adaptive brake control allocation," IEEE Trans. Control System Technology, vol. 18, pp. 545-558, May 2010.

[4] T. K. Podder, and N. Sarkar, "Fault-tolerant control of an autonomous underwater vehicle under thruster redundancy," Robotics and Autonomous Systems, vol. 34, pp. 39-52, Jan. 2001.

[5] I. Sadeghzadeh, A. Chamseddine, Y. Zhang, and D. Theilliol, "Control allocation and re-Allocation for a modified quadrotor helicopter against actuator faults," 8th IFAC Symposium on Fault Detection, Supervision and Safety of Technical Processes, vol. 8, pp. 247-252, Aug. 2012.

[6] A. Casavola, and E. Garone, "Fault-tolerant adaptive control allocation schemes for overactuated systems," Int. J. Robust Nonlinear Sys., vol. 20, pp. 1958-1980, Jan. 2010.

[7] C. Liu,B. Jiang,X. Song, and S. Zhang, "Fault-tolerant control allocation for over-actuated discrete-time systems," J. Franklin Institute, vol. 352, pp. 2297-2313, June 2015.

[8] M. Wang, J. Yang, G. Qin, and Y. Yan, "Adaptive fault-tolerant control with control allocation for flight systems with severe actuator failures and input saturation," American Control Conference (ACC), pp. 5134 5139, June 2013.

[9] C. Liu, X. Zhu, and B. Jiang, "Fault tolerant control based on adaptive control allocation with a multiple effectors aircraft application," American Control Conference (ACC), pp. 2533-2538, June 2012.

[10] D. B. Doman, and A. D. Ngo, "Dynamic inversion-based adaptive/reconfigurable control of the X-33 on ascent," J. Guidance, Control and Dynamics, vol. 25, pp. 275-284, April 2002.

[11] B. Reish, and G. Chowdhary, "Concurrent learning adaptive control in presence of uncertain control allocation matrix," Guidance, Navigation and Control and Co-located Conferences, Aug. 2013.

[12] Y. Liu, and G. Crespo, "Adaptive control allocation in the presence of actuator failures," Journal of Control Science and Engineering, vol. 2012, 2012. 
[13] Y. Liu, X. Tang, G. Tao, and S. M. Joshi, "Adaptive compensation of aircraft actuation failures using an engine differential model," IEEE Trans. Control Syst. Technol., vol. 16, pp. 971-982, 2008.

[14] J. B. Davidson, F. J. Lallman, and W. T. Bundick, "Real-time adaptive control allocation applied to a high performance aircraft," 5th SIAM Conference on Control and Its Applications, 2001.

[15] A. Cristofaro, and T. A. Johansen, "Fault tolerant control allocation using unknown input observers," Automatica, vol. 50, pp. 1891-1897, 2014.

[16] H. Alwi, and C. Edwards, "Fault tolerant control using sliding modes with on-line control allocation," Automatica, vol. 44, pp. 1859-1866, 2008.

[17] T. E. Gibson, "Closed-loop reference model adaptive control: with application to very flexible aircraft," Ph.D. dissertation, Dept. Elect. Eng., M.I.T., Cambridge, MA, 2014.

[18] J. J. E. Slotine, and W. Li, Applied Nonlinear Control. Prentice-Hall, 1991.

[19] Y. Yildiz, and I. V. Kolmanovsky, "A control allocation technique to recover from pilot-induced oscillations (CAPIO) due to actuator rate limiting," American Control Conference (ACC), pp. 516-523, 2010.

[20] O. Harkegard, and S. T. Glad, "Resolving actuator redundancy Optimal control vs. control allocation," Automatica, vol. 41, pp. 137144, 2005.

[21] E. Lavretsky, and T. E. Gibson, "Projection operator in adaptive systems," arXiv ePrints, arXiv:1112.4232, 2011.

[22] T. E. Gibson, A. M. Annaswamy, and E. Lavretsky, "Adaptive systems with closed-loop reference models: stability, robustness and transient performance," arXiv ePrints, arXiv:1201.4897, 2012. 